\title{
ELT Pre-Focal Station NGS Sensor Arm
}

\author{
P.La Penna*,a, E. Brunetto, A. Förster ${ }^{\mathrm{a}}$, C.Frank ${ }^{\mathrm{a}}$, I.Guidolin ${ }^{\mathrm{a}}$, S.Guisard ${ }^{\mathrm{a}}$, P.Hammesley ${ }^{\mathrm{a}}$, \\ R.Holzlohner ${ }^{\mathrm{a}}$, P.Jolley ${ }^{\mathrm{a}}$, J.Kosmalski ${ }^{\mathrm{a}}$, U.Lampater ${ }^{\mathrm{a}}$, S.Lewis ${ }^{\mathrm{a}}$, E.Marchetti ${ }^{\mathrm{a}}$
}

aEuropean Southern Observatory; Karl-Schwarzschild-Strasse 2; D-85748 Garching; Germany

\begin{abstract}
Each Nasmyth platforms of the ELT will host one Prefocal Station, which main function will be to provide a first control of the telescope shape, before delivering the light wavefront to the instruments. Besides providing three Nasmyth focal planes and one Coudé focus, each PFS will include three Natural Guide Star Sensor Arms, rotating on an adapter, to support the low order optimization and control of the telescope shape in seeing limited image quality. Each Sensor Arm will include an image channel, to perform guiding and telescope tip-tilt tracking, and a wavefront sensor, to correct higher order modes. In this paper an opto-mechanical concept of the Sensor Arm is reported.
\end{abstract}

Keywords: ELT, Prefocal Station, Telescope Control, Telescope Guiding, Wavefront Control, Adaptive Optics.

\section{INTRODUCTION}

On each ELT Nasmyth platform a Prefocal Station (PFS) unit will be installed. Besides distributing the telescope beam between different focal stations (three Nasmyth Foci and the Coudé train), the PFS will include a rotating adapter supporting three Natural Guide Star (NGS) Sensor Arms (SA), used as guide probes kept in fixed positions with respect to the telescope focal plane and the sky. The function of these three SA will be supporting the first shaping and control of the telescope, to provide at its exit a seeing limited wavefront. In an opto-mechanical concept developed by ESO, at the top of each SA a pick-off mirror (POM) located $1440 \mathrm{~mm}$ ahead of the natural (f/17.7) focus of the telescope deflects the NGS light to an optical probe system mounted at the base of the arm, rotating on the adapter to follow the NGS natural rotation. Each NGS probe accommodates a 20-arcsecond field of view for acquisition and an over 4-arcsecond stop for the selection of the star whose light will be sent to the wavefront sensor, with the goal of providing a first telescope control and a corrected wavefront to be delivered to the instruments. The NGS probes can track differentially across the field of view following moving targets.

\section{PREFOCAL SENSOR ARM FUNCTION}

The three SA will support telescope acquisition, guiding, seeing limited operation and low order optimization. Low order optimization will be obtained applying an adaptive optics correction, which performances will not go beyond what is needed to achieve seeing limited operation, and without using any laser guide star. Each arm will pick off a different NGS in the telescope field between 2.5 and 5 amin (radial), leaving the field inside 2.5 amin fully unvignetted. NGS field for guiding will be 20 asec (diameter) and 4 asec (diameter) for wavefront correction. The PFS is required to meet all performance over at least $95 \%$ of the sky accessible to the telescope over telescope altitude angles from +30 degrees to +88.5 degrees and 360 degrees in azimuth. Computations show that, for three stars configuration, this can be obtained with at least $m_{R}=18$ and acquisition times up to 1-10 sec.

Specifications for guiding (with low order optimization running, seeing better than 1 arcsec and NGS brighter than $m_{R}=15$ ) provide for a guiding measurement error of 150 mas rms at low frequency $(0.3-20 \mathrm{mHz})$, and of the order of 10 
mas at higher frequencies, at a maximum measurement rate of $500 \mathrm{~Hz}$. Absolute distances between two NGS will be measured with an error smaller than $0.03 \%$.

Low order sensing will run at the maximum rate of $1 \mathrm{~Hz}$. Telescope aberrations will be measured from Zernike mode 4 to 11 , with an error in focus smaller than $750 \mu \mathrm{m}$ ptv, and an error smaller than $125 \mathrm{~nm}$ rms for higher modes. Seeing limited quality sensing will be operated at the maximum rate of $500 \mathrm{~Hz}$. The guiding measurement error will be smaller than 150 mas rms in the frequency range of $0.3-20 \mathrm{mHz}$, and about 50 mas above. The telescope wavefront aberrations will be measured up to the Zernike 100, with a wavefront error not exceeding $250 \mathrm{~nm}$ rms.

\section{PREFOCAL STATION CONCEPT}

The PFS is a structure located at the exit of the ELT telescope, on the Nasmyth platform (see Figure 1). The PFS Main System (the part located on the Nasmyth platform) is an approximately 30 ton high-precision opto-mechanical device. It will occupy a volume of up to $5 \mathrm{~m}$ (width) $\times 4,75 \mathrm{~m}$ (depth) $\times 12,5 \mathrm{~m}$ (height). Part of the light can be sent downwards to another unit (not described in this paper), dedicated to the phasing of the telescope segmented primary mirror. A first analysis of the mechanical concept of the PFS is outlined in [1].

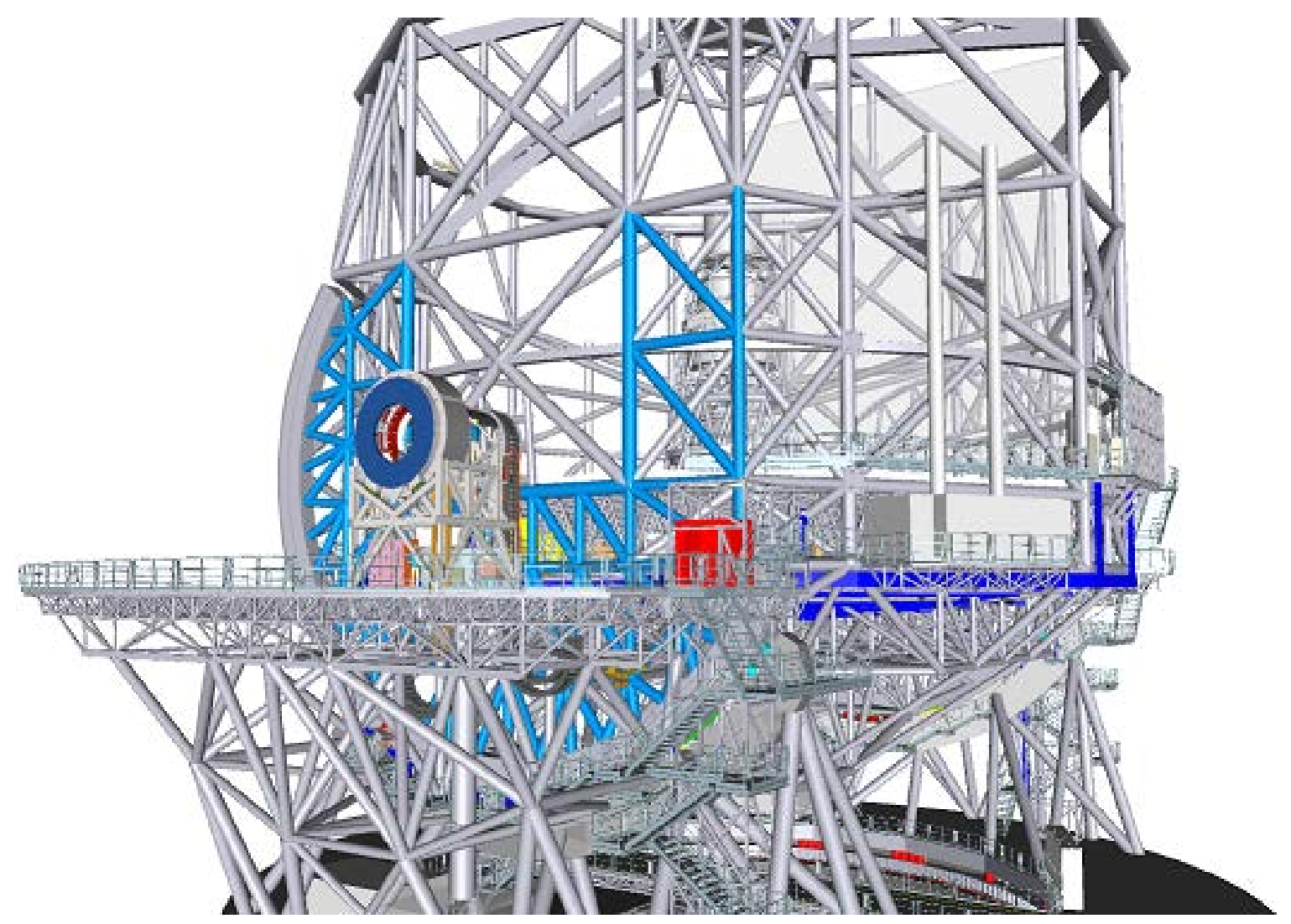

Figure 1: 3D sketch of the Prefocal Station on the ELT Nasmyth platform.

A more developed concept as in [1] is shown in Figure 2. The NGS adapter outer dimensions is $4250 \mathrm{~mm}$ in diameter and $180 \mathrm{~mm}$ in thickness. The present design weight is about $3800 \mathrm{~kg}$.

Three SA are mounted on the adapter, and can rotate to follow the sky. Each SA, which weight is about $270 \mathrm{~kg}$, has an independent rotational and translational degree of freedom, allowing to position its POM to any point in the field of view, except to a certain area in the center of the field. The conceptual design of the Natural Guide star adapter with the SA is shown in Figure 2. The SA must cover the technical field between 2.5 arcmin and 5 arcmin in radius. 

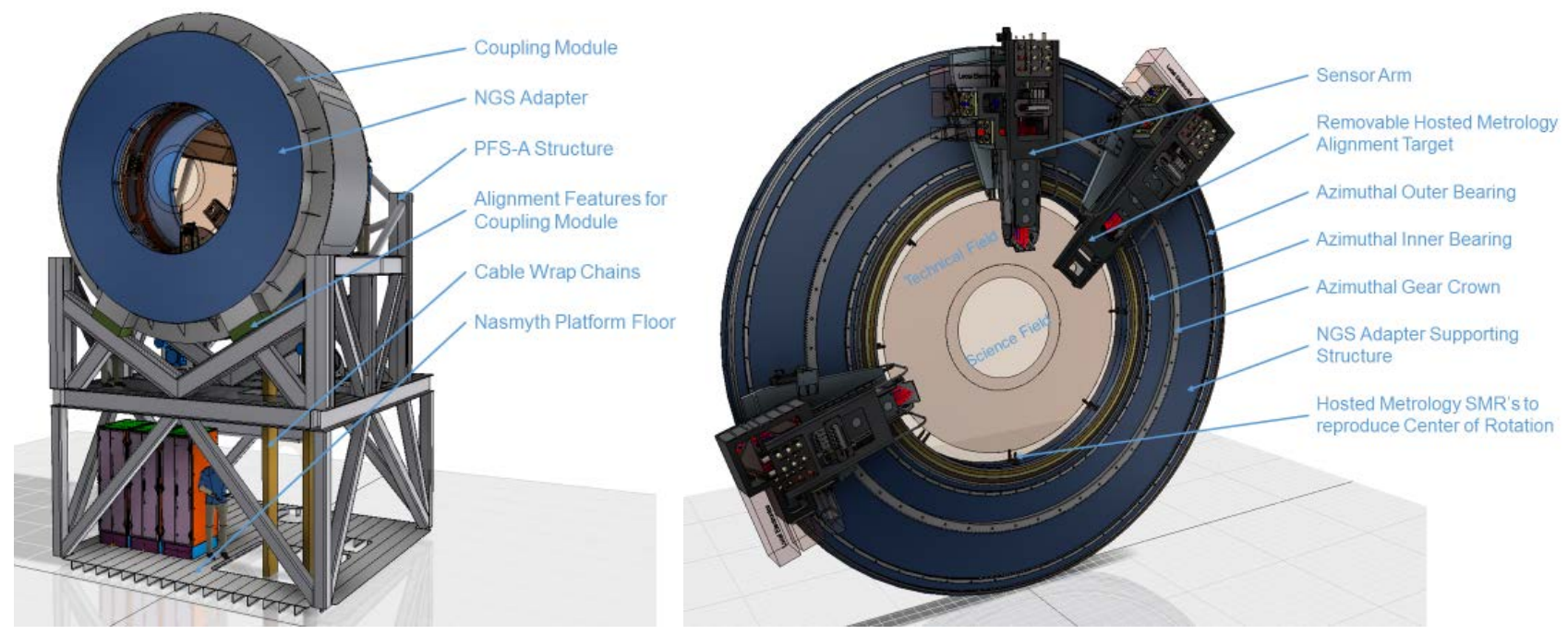

Figure 2: Left: 3D sketch of the PFS on the Nasmyth platform, as seen from the instrument side. Right: PFS adapter rotator, with the three NGS SA.

The SA in the retracted and inserted positions, as shown in Figure 3. The degree of freedom in rotation for a Sensor Arm is not fully independent because it is partially restricted by the presence of the other 2 Sensor Arms: in Figure 2, right, the upper $2 \mathrm{SA}$ are shown in a position with a $45^{\circ}$ separation between the Sensor Arms, which is a requirement for the minimum separation.
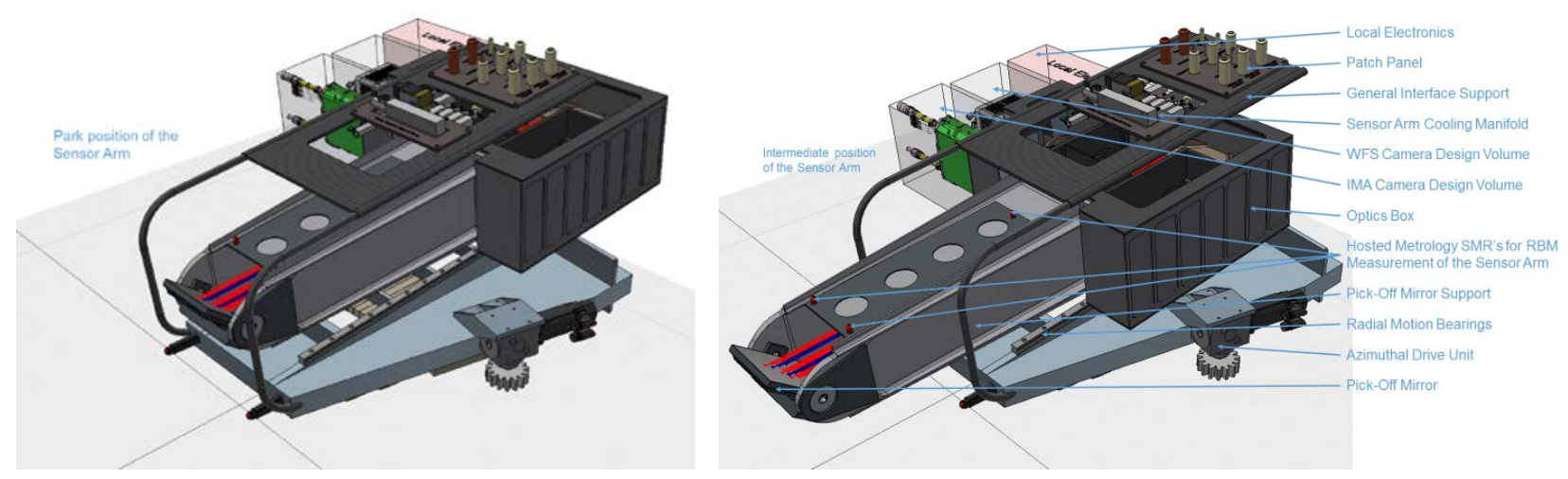

Figure 3: The SA in the retracted (parked) position (left) and in an inserted intermediate position (right).

Each SA will contain many components like optics, actuators, electronics, detectors, calibration sources. The main elements and associated functions of the SA are reported in Table 1.

\section{SENSOR ARM CONCEPTUAL DESIGN}

The opto-mechanical concept of the SA is shown in Figure 6. A POM will pick-off the telescope light (20 asec on-sky field), and direct it to the optical box. The optical box contains the optics for two paths: one, the imaging of tip-tilg (TT) path, is making a focus on the TT detector surface. The other path, the wavefront sensor (WFS) makes the image of the 
telescope pupil on the lenslet array of a WFS. The TT path will receive the light in the wavelength range of 800-1000 $\mathrm{nm}$, while the WFS one will work with the 500-800 nm range. The split of the wavelengths is performed by a dichroic mirror.

\begin{tabular}{|c|c|}
\hline Key Elements & Function \\
\hline Base plate & $\begin{array}{l}\text { Main interface to the NGS adapter supporting structure } \\
\text { Hosts the azimuthal drive units and bearings } \\
\text { Hosts the azimuthal encoder heads to determine the angular position of the Sensor Arm } \\
\text { Hosts the radial encoder tape to determine the radial position of the Sensor Arm } \\
\text { Hosts the metrology SMR's to determine the rotation axis }\end{array}$ \\
\hline Linear guiding system & Enables for radial positioning of the probe \\
\hline $\begin{array}{l}\text { Pick-Off Mirror } \\
\text { support }\end{array}$ & $\begin{array}{l}\text { Hosts the Pick-Off Mirror } \\
\text { Hosts the Flexure bearings of the Pick-Off Mirror } \\
\text { Hosts the actuator to adjust the tilting angle of the Pick-Off Mirror } \\
\text { Provides stiff support for the Pick-Off Mirror }\end{array}$ \\
\hline Pick-Off Mirror & Captures a certain field and to send the light to the optics box \\
\hline Optics Box & $\begin{array}{l}\text { Hosts all required optomechanical functions, to enable analysis for wave front control and } \\
\text { guiding } \\
\text { Provides interface to the WFS and Imaging cameras } \\
\text { Hosts the calibration system to calibrate the detectors and targets } \\
\text { Hosts the field masks } \\
\text { Hosts various shutoff devices to isolate the detectors }\end{array}$ \\
\hline $\begin{array}{l}\text { General interface } \\
\text { support }\end{array}$ & $\begin{array}{l}\text { Hosts the cooling manifold } \\
\text { Hosts the patch panel (I/O) signals } \\
\text { Hosts the power supplies of the WFS and the Imaging cameras }\end{array}$ \\
\hline WFS Camera & Hosts the detector system providing WFS data to the telescopes control system \\
\hline Imaging Camera & $\begin{array}{l}\text { Hosts the detector system providing image data to the telescopes control system, required } \\
\text { for guiding }\end{array}$ \\
\hline Local Electronics & $\begin{array}{l}\text { Hosts all required electrical systems to control the optomechanical functions } \\
\text { Provides a temperature controlled environment for the electrical systems }\end{array}$ \\
\hline
\end{tabular}

Table 1: Breakdown of parts and functions of the SA.

For the WFS (WFS Camera) a new detector currently in development will be used. The detector will have $800 \times 800$ pixels, each of $24 \mu \mathrm{m}$ side, for a total detector area lateral dimension of $19.2 \mathrm{~mm}$. For the TT path (Imaging Camera) it is planned to use a deep depletion CCD 220 camera [2], which detector is more sensitive in the red wavelength side. The CCD 220 has $240 \times 240$ pixels, each with $24 \mu \mathrm{m}$ side, for a total detector area of $5.76 \mathrm{~mm}$. The optical design is driven by the detector specifications. In the TT path the whole 20 asec ( \pm 10 asec radius) field must be contained on the detector area, this leading to a focal surface diameter of $5.76 \mathrm{~mm}$ and a pixel scale of $0.083 \mathrm{asec} /$ pixel. On the WFS path the pupil must be about $10 \%$ smaller than the detector area, this leading to imaging a telescope pupil of about $17 \mathrm{~mm}$ at the exit of the SA optical box.

The NGS is picked off by the POM at the extremity of the arm. Since the output of ELT is not telecentric and the focal plane has a certain curvature $(9884.16 \mathrm{~mm})$, and since the insertion movement of the arm inside the pick-off field is along the line of the linear stage, the angle and the focus of the picked NGS is not the same in the whole field. For this reason, the POM must be tilted (by about \pm 0.2 degrees), and a focus mechanism is needed to keep the NGS focused on the detector if the picked-off radial field is changed. The POM tilt is performed by mounting the POM mirror (a rectangle of about $250 \mathrm{~mm} \times 150 \mathrm{~mm}$ sides) on a tilting mechanism.

The refocus of the NGS is performed using a trombone stage inside the optical box, an element consisting in a block including two plane mirrors (about $140 \mathrm{~mm}$ diameter), at $45^{\circ}$ one with respect to the other. The necessary range to compensate for the ELT focal plane curvature is smaller than $\pm 4 \mathrm{~mm}$, which corresponds to a trombone displacement of $\pm 2 \mathrm{~mm}$, this being sufficient to keep the ELT focal plane inside the optical box always at the same position. In addition to 
this compensation, the SA shall accommodate an additional change of BFL of the telescope by maximum $\pm 50 \mathrm{~mm}$. It achieves it by moving the trombone by about $\pm 25 \mathrm{~mm}$ along the optical path.
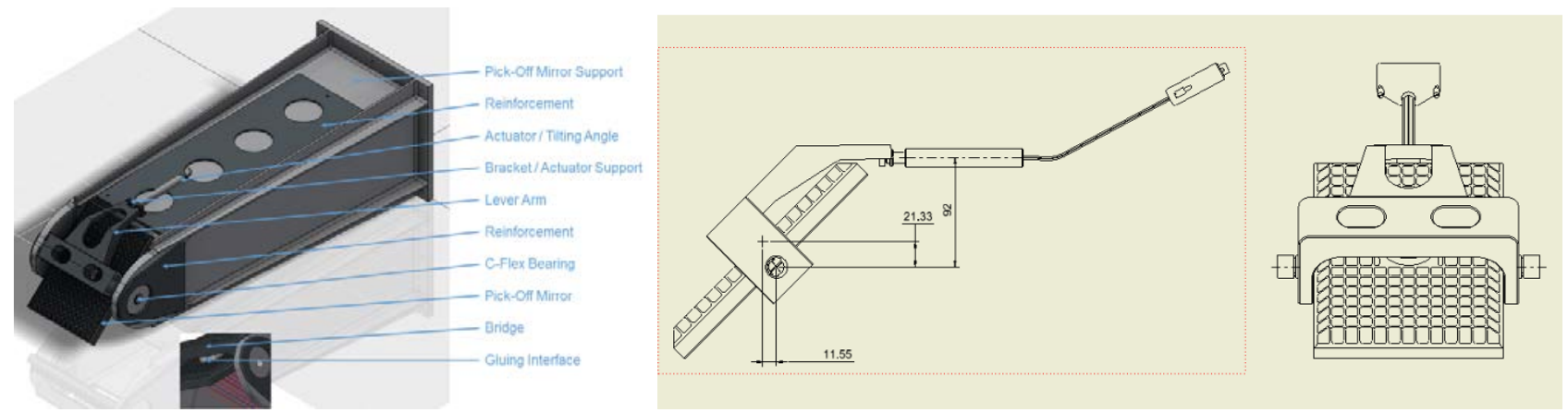

Figure 4: Left: the arm with the POM. Right: mechanical drawing of the POM mount.

A large dichroic mirror (about 110mm diameter) splits the light into the two paths (TT and WFS): the WFS receives the reflected wavelength range between $500 \mathrm{~nm}-800 \mathrm{~nm}$, the TT gets the range between $800 \mathrm{~nm}$ and $950 \mathrm{~nm}$. A field stop at the level of the Intermediate ELT Focal Plane stops the fields outside 10asec (radial). On the WFS path, right after the reflection by the dichroic, another stop lets pass unvignetted the 2asec (radial) field. Fields up to about 4asec are transmitted partially vignetted.
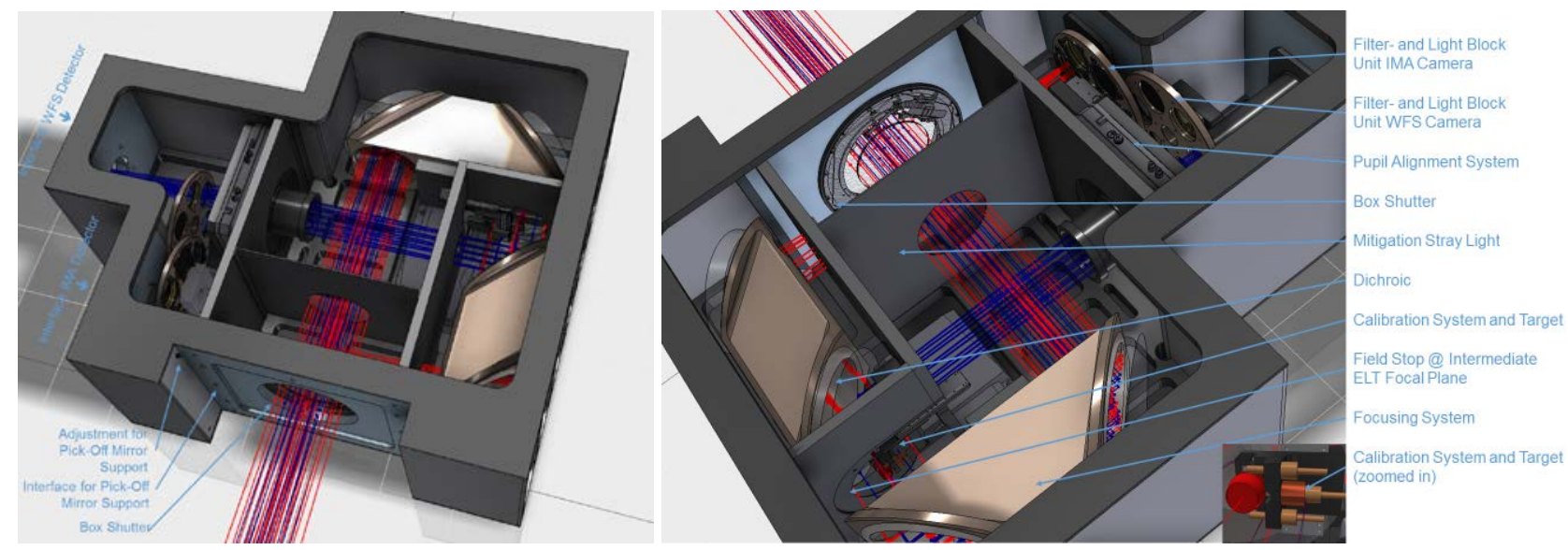

Figure 5: CAD model of the Optical Box of the SA: the trombone (Focusing System) keeps the focal plane of ELT in the same position inside the Optical Box (Intermediate ELT Focal Plane in the figure). A calibration unit system can be inserted on the intermediate ELT focal plane. The pupil reimaging lens is mounted on lateral translation stages, which allows to center the pupil on the detector (Pupil Alignment System).

\subsection{Imaging path}

The imaging path will produce a focal spot on the detector. This spot will be kept on the central reference pixel by correcting the telescope angle during tracking. Three NGS are used, so that in addition to tilts also rotations of the field can be corrected. The design diffraction limited SA on-axis spot, besides exhibiting the sufficient mechanical stability to be a stable reference for the guiding, would be smaller than the best expected seeing conditions. 


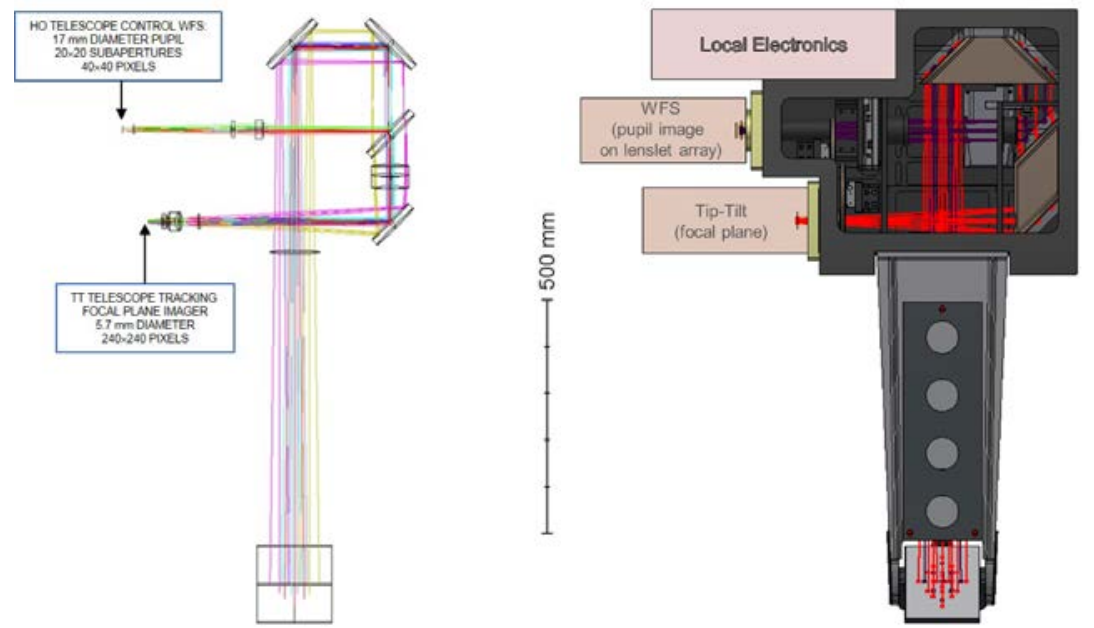

Figure 6: Left: optical drawing of the SA. Right: optomechanical concept.

The main design drivers for the imaging path are the following:

Pixel scale (Keeping one pixel margin):

Seeing conditions:

Position stability:

Radius at $80 \%$ EE (on axis):

Plate scale stability:

Plate scale difference between $\mathrm{X}$ and $\mathrm{Y}$ :
20 asec/238=84 mas/pixel

$>0.4$ asec (Seeing $\geq 4.76$ pixels)

$\leq 30$ mas rms $(\leq 0.36$ pixels $)$

$\leq$ seeing 0.4 " $=4.8$ pixels (diameter)

$<1 \%$

$<0.3 \%$

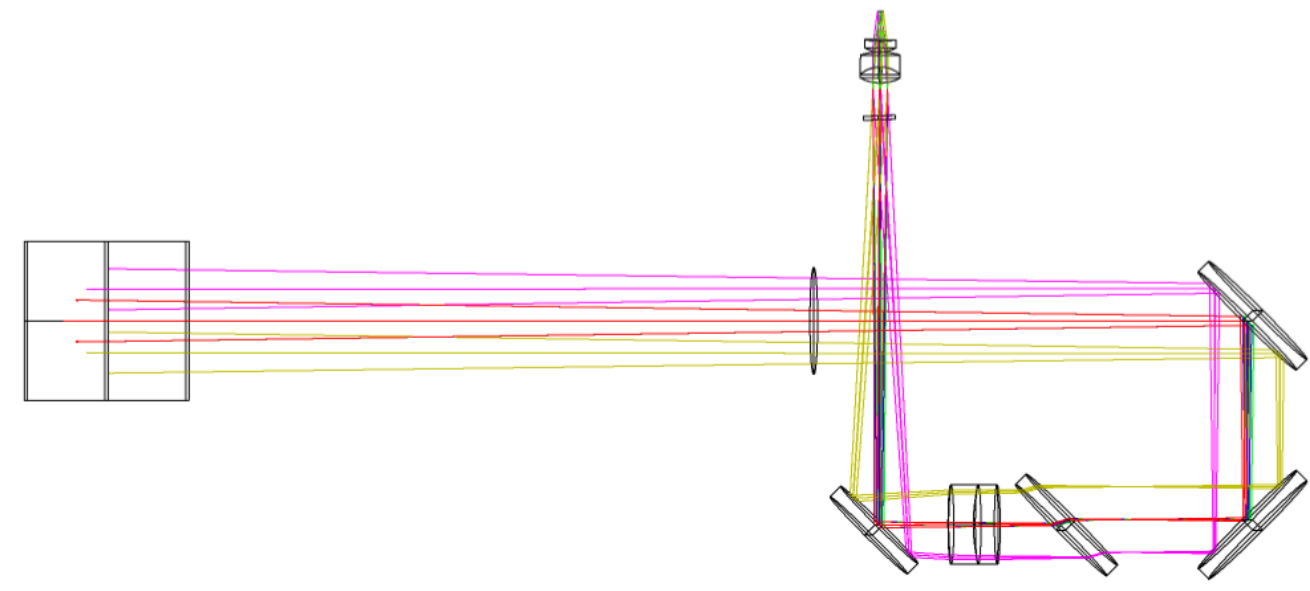

Figure 7: Optical layout of the SA imaging path. The light is reflected by the POM and by the two mirrors of the trombone. On the ELT focal plane, after the trombone, a field stop lets only 20asec field pass. The wavelength range 800-950nm is then transmitted by a dichroic, a doublet lens collimates the light close to a triplet that creates a focal plane having the diameter of $5.7 \mathrm{~mm}$ on the TT detector. 

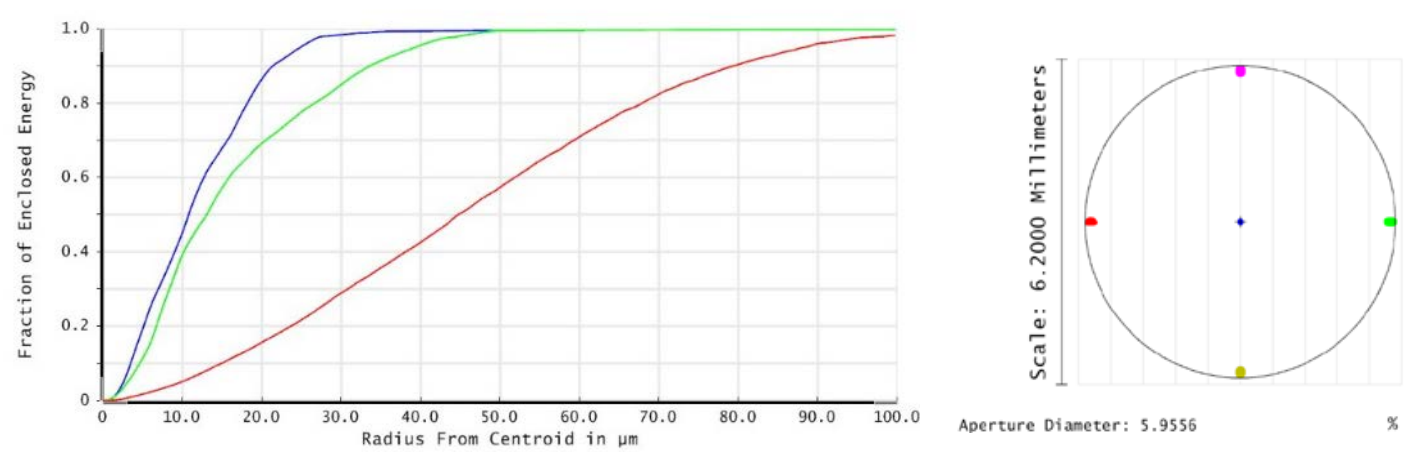

Figure 8: Left: Polycromatic Encircled Energy plot for the SA centered on the 3.75amin on-sky field (central accessible field): Blue: 3.75amin; Green: 3.75amin + 5asec; Red: 3.75amin +10asec. Right: footprint on the detector plane. The central spot (on axis) diameter is smaller than 2 pixels. Results are similar for the whole SA accessible field.

The linearity of the pixel scale is shown in Figure 9. The deviation of the plate scale with respect to the linear 0.286 $\mathrm{mm} / \mathrm{sec}$ is always smaller than $60 \mu \mathrm{m}(0.2 \%)$.
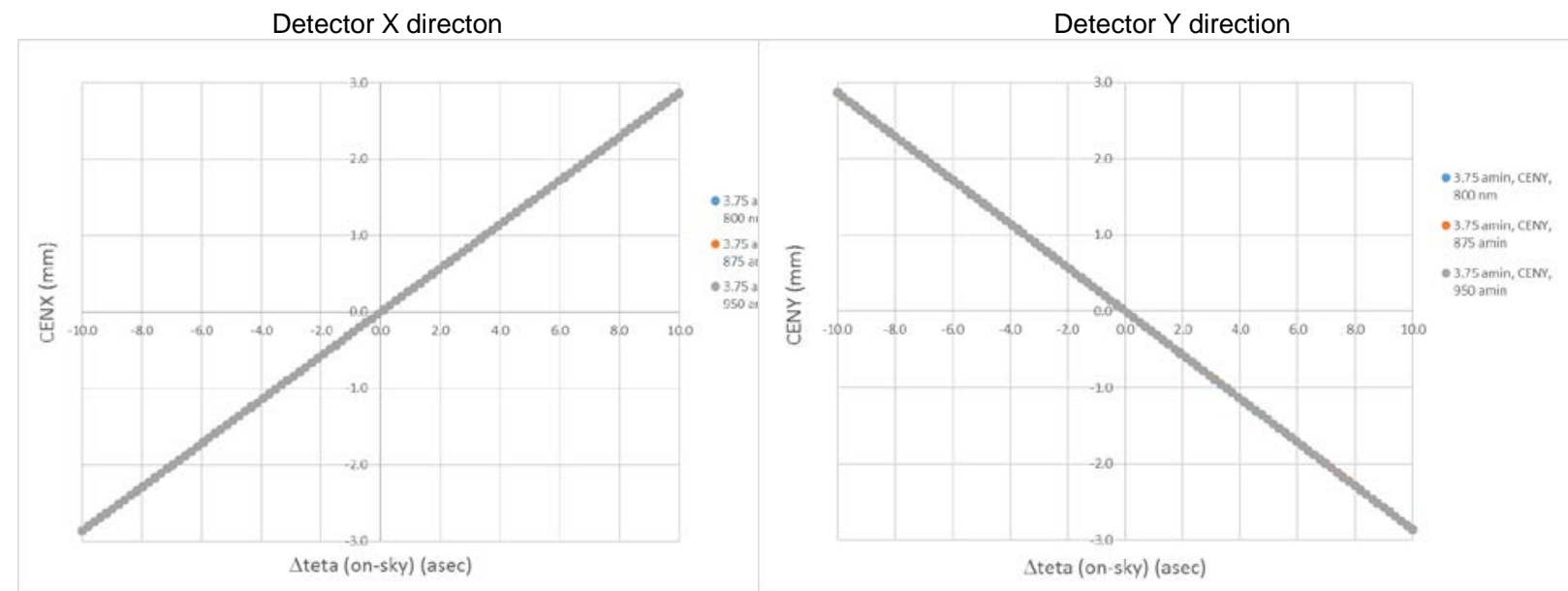

Figure 9: pixel scale linearity: for the SA centered on 3.75amin on-sky, spot position on detector when the field position is changed by \pm 10 asec with respect to the POM centre position. The displacement is well linear, with a plate scale of $2.86 \mathrm{~mm} / 10$ asec (11 pixels/asec, with a pixel of $24 \mu \mathrm{m}$ ). The deviation of the plate scale with respect to the linear $0.286 \mathrm{~mm} /$ asec is always smaller than $60 \mu \mathrm{m}(0.2 \%)$.

\subsection{WFS path}

The main design drivers for the optical design of the WFS path are:

Input field: $\quad 0, \pm 2$ asec (fully unvignetted)

Wavelength: $\quad 500-800 \mathrm{~nm}$

Pupil diameter: $17 \mathrm{~mm}$ (708 pixels)

Pupil blur: $\quad<3 \%$

WFE: $\quad<90 \mathrm{~nm}$ rms (focus)

$<125 \mathrm{~nm}$ rms (modes $>\mathrm{Z}=4$ to $\mathrm{Z}=37$ ) 
The pupil will be smaller than the detector area, in order to leave margin (something more than 10\%) for the position wandering of the pupil. In addition to this margin, the pupil reimaging lens that makes the image of the telescope image in front of the detector can be shifted laterally, such that the pupil position can be adjusted by about $\pm 2 \mathrm{~mm}$ in $\mathrm{X}$ and $\mathrm{Y}$. The pupil is imaged on a lenslet array (LA) placed fixed in front of the detector. The LA will have 20 subapertures, each corresponding to 40 pixels on the detector ( $960 \mu \mathrm{m}$ side). The imaged pupil will therefore cover 18 subapertures. With a LA dimensioned to a pixel scale of 0.3 asec/pixel (in order to sufficiently sample even the best expected seeing 0.4 asec), each subaperture will have a field of 12 asec $( \pm 6$ asec).

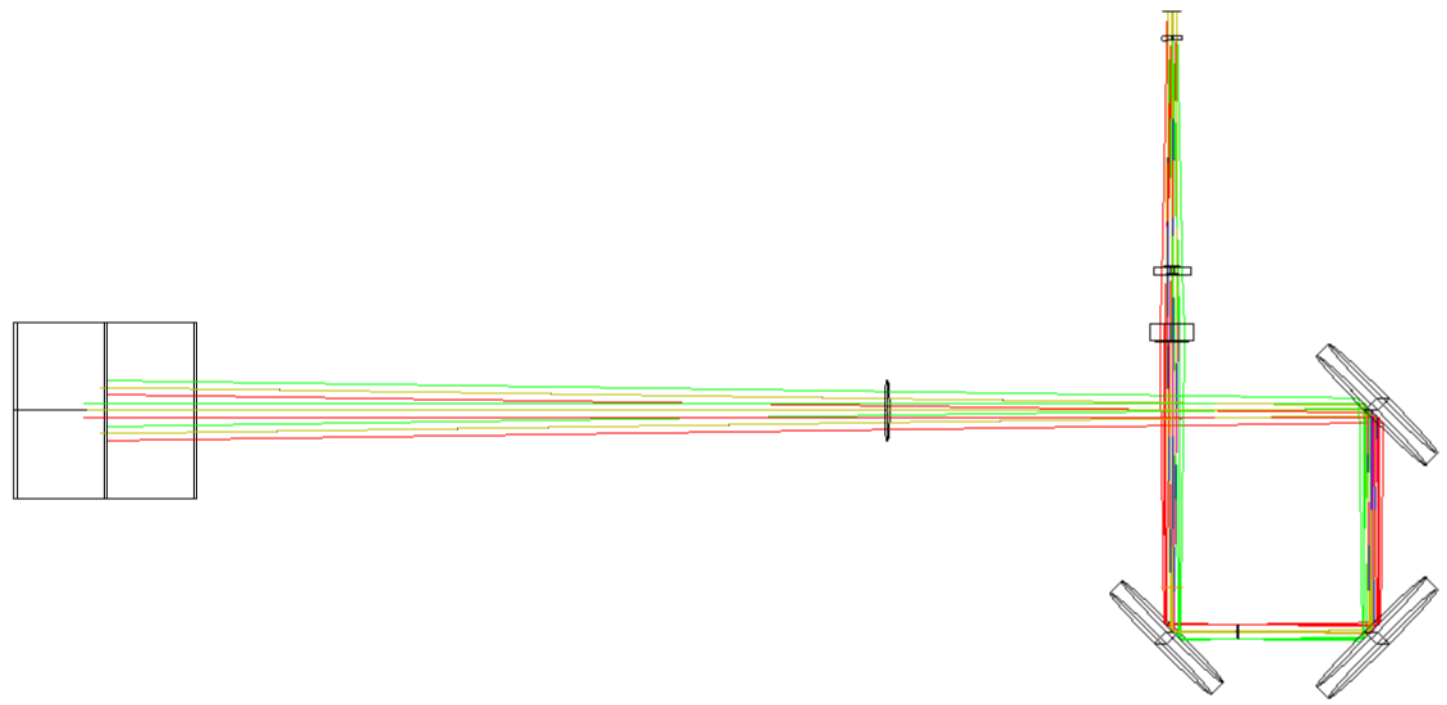

Figure 10: Optical scheme of the WFS path of the SA. The pupil reimaging lens can be moved laterally to adjust the centering of the pupil on the detector.

The characteristics of the pupil shape and wavefront error are shown in Figure 11. The maximum ptv blur never exceeds $7 \%$ of a subaperture (at the edge of the pupil, less than $5 \%$ close to the center). For the on-axis field, the maximum (chromatic) blur is smaller than 3\%. The total wavefront error (that is, including all the modes, also focus) is basically diffraction limited for all the field in the central wavelength range, and it never exceeds $70 \mathrm{~nm}$ rms.
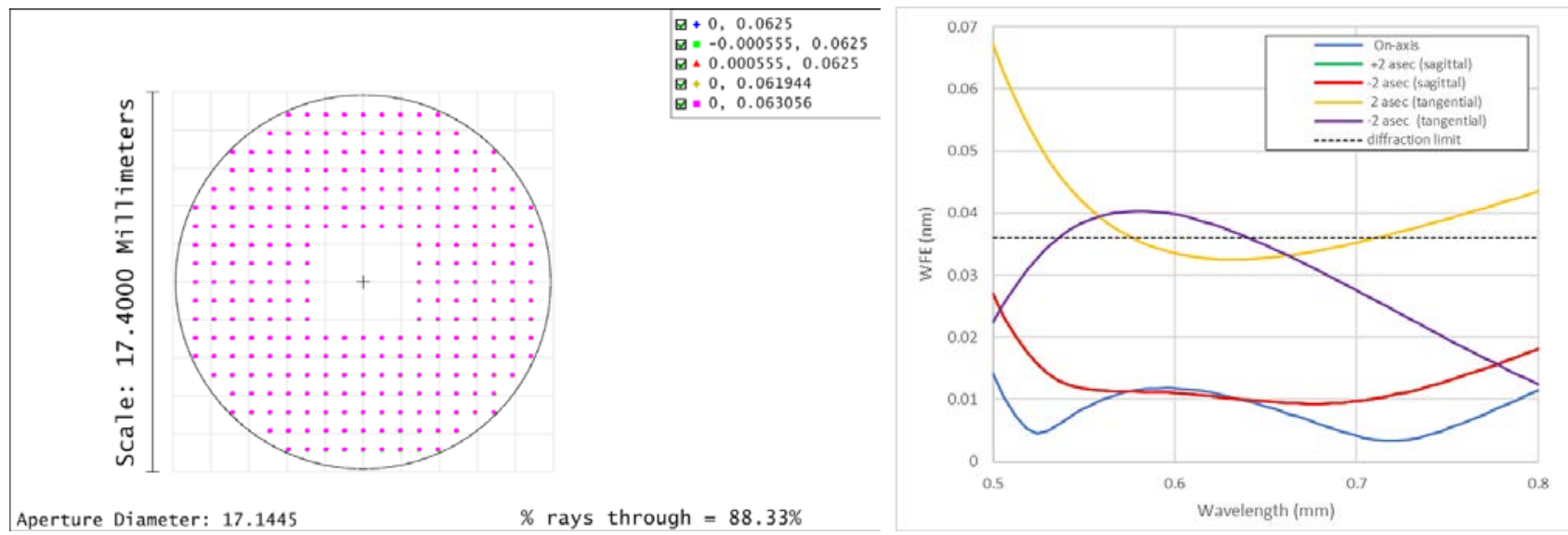

Figure 11: Left: footprint of the pupil on the lenslet array plane of the WFS detector (SA centered on 3.75amin on-sky). The maximum ptv blur on the pupil, on the whole wavelength range $(500-800 \mathrm{~nm})$ and unvignetted field ( \pm 2 asec on-sky) is $70 \mu \mathrm{m}$ (7\% of a subaperture), less than 3\% on axis. Right: in the same conditions, plot of the wavefront error: mostly diffraction limited, is always less than $70 \mathrm{~nm}$ rms. 


\section{CONCLUSION}

ELT will host on the Nasmyth platform a PFS including three rotating NGS SA, which scope is to perform the first guiding and control of the telescope shape. The extensive opto-mechanical design, developed at ESO and outlined in this paper, meets the tight specifications required for this system and demonstrates its feasibility. The call for tender for the industrial contract has been launched at end of July 2017.

\section{REFERENCES}

[1] Jolley P., et al., "Prefocal Station mechanical design concept study for the E-ELT", Proc. of SPIE Vol. 9906 99062H-1 (2016).

[2] Reyes, J. et al., “An overview of the ESO adaptive optics wavefront sensing camera”, Proc. SPIE, 8447, 237 (2012). 